\begin{tabular}{|c|l|}
\hline Title & I sotope scattering of phonons in superlattices \\
\hline Author(s) & Tamura, Shin-ichiro \\
\hline Citation & $\begin{array}{l}\text { Physical Review B, 56/19), 12440-12445 } \\
\text { https://doi.org/10.1103/PhysRevB.56.12440 }\end{array}$ \\
\hline Issue Date & 1997-11-15 \\
\hline Doc URL & http://hdl.handle.net/2115/5967 \\
\hline Rights & Copyright $\odot$ 1997 A merican Physical Society \\
\hline Type & article (author version) \\
\hline File Information & PRB56-19.pdf \\
\hline
\end{tabular}

Instructions for use 


\title{
Isotope scattering of phonons in superlattices
}

\author{
Shin-ichiro Tamura \\ Department of Applied Physics, Hokkaido University, Sapporo 060, Japan
}

(Received 23 May 1997; revised manuscript received 4 August 1997)

\begin{abstract}
The elastic scattering rate of acoustic phonons in periodic superlattices due to isotopic atoms is calculated in the isotropic, continuum approximation. Unlike the case in bulk solids, the scattering rate depends on both the propagation direction and mode of phonons. Numerical examples are given for AlAs/GaAs and Al/W superlattices for propagation along the normal to the layer interfaces. We find that the scattering of longitudinal phonons is much weaker than that of transverse phonons for wide ranges of frequencies. [S0163-1829(97)03244-X]
\end{abstract}

The propagation and scattering of acoustic phonons in superlattices are of both fundamental and practical interest. An increasing number of semiconductor devices incorporate superlattice structures and the heat generated at an active region of a device is dissipated eventually in the form of acoustic phonons. So far, the basic propagation characteristics of phonons in superlattices have been extensively studied both theoretically and experimentally. ${ }^{1}$ However, studies of the scattering of acoustic phonons in this type of multilayered structure are very limited.

Recently, the thermal conductivity of AlAs/GaAs superlattices has been measured over a wide range of temperatures. ${ }^{2}$ Surprisingly, in the growth direction of the superlattice, the thermal conductivity is reduced by about one order of magnitude relative to that of bulk GaAs. No quantitative theory accounting for this result exists, though the reduction of thermal conductivity is expected by qualitative argument. This is because the folding of phonon dispersion relations associated with the artificial periodicity of a superlattice structure is expected to reduce the group velocity of phonons. In addition, umklapp anharmonic scatterings become possible at energies much smaller than those in a bulk crystal. $^{3}$

In the present work we calculate the isotope scattering rate of acoustic phonons in periodic AlAs/GaAs and also $\mathrm{Al} / \mathrm{W}$ superlattices. ${ }^{4}$ It should be noted that the elements $\mathrm{Al}$ and As are isotopically pure and thus elastic scattering in the former occurs from the mass difference between ${ }^{69} \mathrm{Ga}$ and ${ }^{71} \mathrm{Ga}$ atoms in the GaAs layers and in the latter from the five isotopic atoms ${ }^{180} \mathrm{~W},{ }^{182} \mathrm{~W},{ }^{183} \mathrm{~W},{ }^{184} \mathrm{~W}$, and ${ }^{186} \mathrm{~W}$.

The interaction Hamiltonian $H_{I}$ that describes the elastic scattering of phonons due to mass fluctuation in a layering structure is given by ${ }^{5}$

$$
H_{I}=\int d^{3} x \Delta \rho(\mathbf{x})[\mathbf{P}(\mathbf{x})]^{2} / 2\left[\rho_{0}(z)\right]^{2},
$$

where $\mathbf{P}(\mathbf{x})$ is the momentum operator canonically conjugate to the displacement field of the phonons, $\rho_{0}$ either represents the discontinuous mass density distribution with a periodic parapet profile along the growth direction $z$ (the interfaces are parallel to the $\mathbf{x}_{\|}$plane) or takes the averaged mass density in each constituent layer, and $\Delta \rho$ is the mass density fluctuation around $\rho_{0}$. In a superlattice consisting of two neighboring layers $A$ (thickness $d_{A}$ ) and $B$ (thickness $d_{B}$ ), $\mathbf{P}$ takes the form

$$
\mathbf{P}(\mathbf{x})=-i \sum_{\lambda}\left[\frac{\hbar \omega_{\lambda} \rho_{0}(z)}{2 N D S}\right]^{1 / 2}\left(c_{\lambda}-c_{-\lambda}^{\dagger}\right) \mathbf{W}_{\lambda}(z) e^{i \mathbf{q}\|\cdot \mathbf{x}\|,}
$$

$$
\begin{aligned}
& \mathbf{W}_{\lambda}(z)=\sum_{n} e^{i q_{z} n D}\left\{\Theta(z-n D) \Theta\left(n D+d_{A}-z\right) \mathbf{A}_{\lambda}(z-n D)\right. \\
& \left.+\Theta\left(z-n D-d_{A}\right) \Theta[(n+1) D-z] \mathbf{B}_{\lambda}(z-n D)\right\} \\
& \equiv \sum_{n} e^{i q_{z} n D} \mathbf{u}_{\lambda}(z-n D) \\
& \rho_{0}(z)=\sum_{n}\left\{\rho_{0}^{A} \Theta(z-n D) \Theta\left(n D+d_{A}-z\right)\right. \\
& \left.+\rho_{0}^{B} \Theta\left(z-n D-d_{A}\right) \Theta[(n+1) D-z]\right\}, \\
& \mathbf{A}_{\lambda}(z)=\sum_{l=1}^{3}\left[t_{\lambda, l}^{A} \mathbf{e}_{\lambda, l}^{A} \exp \left(i k_{\lambda, l}^{A} z\right)+r_{\lambda, l}^{A} \widetilde{\mathbf{e}}_{\lambda, l}^{A} \exp \left(-i k_{\lambda, l}^{A} z\right)\right], \\
& \mathbf{B}_{\lambda}(z)=\sum_{l=1}^{3}\left\{t_{\lambda, l}^{B} \mathbf{e}_{\lambda, l}^{B} \exp \left[i k_{\lambda, l}^{B}\left(z-d_{A}\right)\right]\right. \\
& \left.+r_{\lambda, l}^{B} \widetilde{\mathbf{e}}_{\lambda, l}^{B} \exp \left[-i k_{\lambda, l}^{B}\left(z-d_{A}\right)\right]\right\},
\end{aligned}
$$

where $\lambda=\left(\mathbf{q}_{\|}, q_{z}, j, m\right)$ is a set of quantum numbers comprising the wave vector $\mathbf{Q} \equiv\left(\mathbf{q}_{\|}, q_{z}\right)$, mode index $j$, and band index $m$. It should be noted that in general each mode consists of three pairs (indexed by $l$ ) of counterpropagating waves. ${ }^{6}$ However, in the isotropic approximation the two sagittal modes $(j=1,2)$ consist of two pairs of counterpropagating longitudinal $(l=1)$ and transverse $(l=2)$ waves and the horizontally polarized shear mode ( $\mathrm{SH}$ mode, $j=3$ ) consists of a single pair of counterpropagating transverse $(l=3)$ waves. Also in the above equations $N$ is the periodicity number and $S$ is the interface area, $c_{\lambda}$ and $c_{\lambda}^{\dagger}$ are the phonon annihilation and creation operators satisfying $\left[c_{\lambda}, c_{\lambda^{\prime}}^{\dagger}\right]$ $=\delta_{\lambda, \lambda^{\prime}}, D=d_{A}+d_{B}$ is the length of the unit period, $\rho_{0}^{A}\left(\rho_{0}^{B}\right)$ 
is the averaged mass density of the $A(B)$ layer, $\Theta$ is the unit step function, $t_{\lambda, l}$ and $r_{\lambda, l}$ are the transmission and reflection coefficients, $\mathbf{e}_{\lambda, l}$ and $\tilde{\mathbf{e}}_{\lambda, l}$ are the unit polarization vectors, and $k_{\lambda, l}^{I}(I=A, B)$ is the wave number in a layer along the growth direction. It should be remarked here that the wave vector $\mathbf{Q}$ and angular frequency $\omega_{\lambda}$ define the superlattice phonon dispersion, whereas the wave vector $\mathbf{K}_{\lambda, l}^{I} \equiv\left(\mathbf{q}_{\|}, k_{\lambda, l}^{I}\right)$ and $\omega_{\lambda}$ for $I=A$ and $B$ define the bulk phonon dispersion relations of the constituent materials. Equation (2) satisfies Bloch's theorem required for a periodic system. The magnitudes of $t_{\lambda, l}$ and $r_{\lambda, l}$ are normalized according to $\int_{0}^{D}\left|\mathbf{u}_{\lambda}(z)\right|^{2} d z=D$ or, more generally,

$$
\int_{0}^{D}\left[\mathbf{u}_{\mathbf{q}_{\|}, q_{z}, j^{\prime}, m^{\prime}}(z)\right]^{*} \cdot \mathbf{u}_{\mathbf{q}_{\|}, q_{z}, j, m}(z) d z=D \delta_{j^{\prime}, j} \delta_{m^{\prime}, m} .
$$

With these equations the interaction Hamiltonian becomes

$$
H_{I}=\frac{\hbar}{4 L} \sum_{\lambda, \lambda^{\prime}}\left(\omega_{\lambda} \omega_{\lambda^{\prime}}\right)^{1 / 2} c_{\lambda} c_{\lambda^{\prime}}^{\dagger} \Phi_{\lambda,-\lambda^{\prime},-} \mathbf{q}_{\|}+\mathbf{q}^{\prime} \| \text { H.c., }
$$

where $L=N D$ is the length of the superlattice, $-\lambda=\left(-\mathbf{q}_{\|}, q_{z}, j, m\right)$,

$$
\Phi_{\lambda, \lambda^{\prime}, \mathbf{k}_{\|}}=\int d z \frac{\Delta \rho_{\mathbf{k}_{\|}}(z)}{\rho(z)} \mathbf{W}_{\lambda}(z) \cdot \mathbf{W}_{\lambda^{\prime}}(z),
$$

and $\Delta \rho_{\mathbf{k}_{\|}}$is defined by

$$
\Delta \rho(\mathbf{x})=\sum_{\mathbf{k}_{\|}} \Delta \rho_{\mathbf{k}_{\|}}(z) e^{i \mathbf{k}\|\cdot \mathbf{x}\|}
$$

or

$$
\Delta \rho_{\mathbf{k}_{\|}}(z)=\frac{1}{S} \int d^{2} x_{\|} \Delta \rho(\mathbf{x}) e^{-i \mathbf{k}\|\cdot \mathbf{x}\|} .
$$

Applying the golden rule for the transition rates, we obtain the following equation for the deviation $\Delta n_{\lambda}$ of the phonon occupation number from the thermal equilibrium value:

$$
\begin{aligned}
\frac{\Delta \dot{n}_{\lambda}}{\Delta n_{\lambda}} & =-\frac{1}{\tau_{\lambda}} \\
& =-\frac{\pi}{2 L^{2}} \omega_{\lambda}^{2} \sum_{\lambda^{\prime}} \delta\left(\omega_{\lambda}-\omega_{\lambda^{\prime}}\right)\left|\Phi_{\lambda,-\lambda^{\prime},-\mathbf{q}_{\|}+\mathbf{q}_{\|}^{\prime}}\right|^{2} .
\end{aligned}
$$

Now we take the configuration average over mass density fluctuations

$$
\begin{aligned}
&\left\langle\Delta \rho\left(\mathbf{x}_{\|}, z+n D\right) \Delta \rho\left(\mathbf{x}_{\|}^{\prime}, z^{\prime}+n^{\prime} D\right)\right\rangle \overline{\left[\Delta M_{I}\right]^{2}} \\
& \quad=\frac{V_{0}^{I}}{V_{n, n^{\prime}}} \delta\left(\mathbf{x}_{\|}-\mathbf{x}_{\|}^{\prime}\right) \delta\left(z-z^{\prime}\right),
\end{aligned}
$$

where $I=A$ if $0<z<d_{A}, I=B$ if $d_{A}<z<D$, and $V_{0}^{A}$ and $V_{0}^{B}$

\begin{tabular}{|c|c|c|c|c|}
\hline$I$ & $\rho_{0}\left(\mathrm{~cm}^{-3}\right)$ & $v_{l}\left(10^{5} \mathrm{~cm} / \mathrm{s}\right)$ & $v_{t}\left(10^{5} \mathrm{~cm} / \mathrm{s}\right)$ & $g_{I}$ \\
\hline $\mathrm{Al}$ & 2.73 & 6.25 & 3.21 & 0 \\
\hline W & 19.32 & 5.21 & 2.88 & $7.01 \times 10^{-5}$ \\
\hline GaAs & 5.32 & 5.03 & 3.03 & $4.58 \times 10^{-5}$ \\
\hline AlAs & 3.76 & 5.98 & 3.60 & 0 \\
\hline
\end{tabular}
are the unit-cell volumes of $A$ and $B$ materials. In Eq. (13)

$$
\overline{\left[\Delta M_{I}\right]^{2}}=\sum_{i=1}^{n_{I}} f_{I, i}\left(M_{I, i}-\bar{M}_{I}\right)^{2}, \quad I=A, B
$$

TABLE I. Employed numerical values of the averaged mass density $\rho_{0}$, bulk longitudinal and transverse sound velocities $v_{l}$ and $v_{t}$, and $g_{I}$ measuring the mass-density fluctuation for each material. For GaAs the value of $g_{G a}\left(\bar{M}_{G a}\right)^{2} /\left(\bar{M}_{G a}+\bar{M}_{A s}\right)^{2}$ is given in the column of $g_{I}$.

measures the mass fluctuation due to the isotope distributions, where $n_{I}$ is the number of isotopes, $f_{I, i}$ and $M_{I, i}$ are the fraction and mass of $I$ material due to $i$ th isotope, and $\bar{M}_{I}$ is the average mass, i.e., $\bar{M}_{I}=\rho_{0}^{I} V_{0}^{I}$. With the above averaging procedure we have

$$
\begin{aligned}
\left|\Phi_{\lambda,-\lambda^{\prime},-\mathbf{q}_{\|}+\mathbf{q}^{\prime} \|}\right|^{2}= & \frac{N}{S}\left\{V_{0}^{A} g_{A} \int_{0}^{d_{A}}\left|\mathbf{A}_{\lambda}(z) \cdot \mathbf{A}_{\lambda^{\prime}}^{*}(z)\right|^{2} d z\right. \\
& \left.+V_{0}^{B} g_{B} \int_{d_{A}}^{D}\left|\mathbf{B}_{\lambda}(z) \cdot \mathbf{B}_{\lambda^{\prime}}^{*}(z)\right|^{2} d z\right\},
\end{aligned}
$$

where $g_{I}=\overline{\left[\Delta M_{I}\right]^{2}} /\left(\bar{M}_{I}\right)^{2}$.

For a numerical calculation we consider the $\mathrm{AlAs} / \mathrm{GaAs}$ and $\mathrm{Al} / \mathrm{W}$ superlattices, whose phonon band structures have been studied extensively. ${ }^{7}$ Specifically, the Al/W superlattice exhibits large-frequency gaps in its phonon dispersion relations due to large acoustic mismatch between the constituent materials (see Table I). Although aluminum $\left(\bar{M}_{A l}=26.98\right.$ amu) and arsenic $\left(\bar{M}_{A s}=74.92 \mathrm{amu}\right)$ are isotopically pure, natural gallium consists of two isotopes ${ }^{69} \mathrm{Ga}$ and ${ }^{71} \mathrm{Ga}$ (the relative abundances are 0.602 and $0.398, \bar{M}_{G a}=69.72 \mathrm{amu}$, and $\left.g_{G a}=1.97 \times 10^{-4}\right)$. Hence the mass-difference scattering in the GaAs superlattice occurs only in the GaAs layers with $g_{G a A s}=g_{G a}\left(\bar{M}_{G a}\right)^{2} /\left(\bar{M}_{G a}+\bar{M}_{A s}\right)^{2}=4.58 \times 10^{-5}$. A similar situation holds for the $\mathrm{Al} / \mathrm{W}$ superlattice, where tungsten consists of five isotopes $\left(\bar{M}_{W}=183.8\right.$ amu and $g_{W}=7.01$ $\times 10^{-5}$ ). Thus the following formula is applied to calculate numerically the isotope scattering rate in these superlattices with $A$ the GaAs or tungsten:

$$
\tau_{\lambda}^{-1}=\frac{N V_{0}^{A} g_{A} \omega_{\lambda}^{2}}{16 \pi^{2} L} \sum_{j^{\prime}} \int_{\omega_{\lambda^{\prime}}=\omega_{\lambda}} \frac{d^{2} q_{\|}^{\prime}}{v_{\lambda^{\prime}}} \int_{0}^{d_{A}}\left|\mathbf{A}_{\lambda}(z) \cdot \mathbf{A}_{\lambda^{\prime}}^{*}(z)\right|^{2} d z,
$$

with $v_{\lambda}=\left|d \omega_{\lambda} / d q_{z}\right|$, or, more explicitly,

$$
\tau_{\lambda}^{-1}=\frac{V_{0}^{A} d_{A}}{4 \pi D^{5}} g_{A} v_{0} I_{A}\left(\omega_{\lambda}\right)
$$

where 

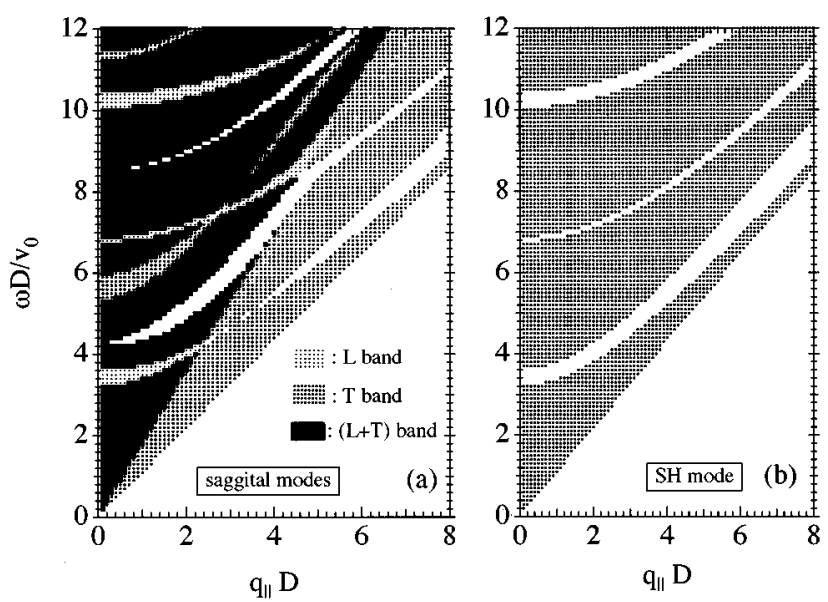

FIG. 1. Band structures of phonons in the AlAs/GaAs superlattice with $d_{\text {GaAs }}=d_{A l A s}$. (a) Frequency bands of sagittal modes. The simultaneous bands of the longitudinal $(L)$ and transverse $(T)$ modes are filled in black. (b) Frequency bands of the SH mode. The numerical values of the sound velocities used are $5.03 \times 10^{5}$ and $5.98 \times 10^{5} \mathrm{~cm} \mathrm{~s}^{-1}$ ( $L$ modes) and $3.03 \times 10^{5}$ and $3.60 \times 10^{5} \mathrm{~cm} \mathrm{~s}^{-1}$ ( $T$ modes) for GaAs and AlAs, respectively, and the mass densities are $5.32 \mathrm{~g} \mathrm{~cm}^{-3}$ for GaAs and $3.76 \mathrm{~g} \mathrm{~cm}^{-3}$ for AlAs. $v_{0}$ is the transverse sound velocity $\left(3.03 \times 10^{5} \mathrm{~cm} \mathrm{~s}^{-1}\right)$ in GaAs.

$$
\begin{aligned}
I_{A}\left(\omega_{\lambda}\right)= & \left(\frac{\omega_{\lambda} D}{v_{0}}\right)^{2} \\
& \times \sum_{j^{\prime}} \int_{\omega_{\lambda^{\prime}}=\omega_{\lambda}} \frac{d^{2}\left(q_{\|}^{\prime} D\right)}{2 \pi}\left|\frac{d\left(\omega_{\lambda^{\prime}} D / v_{0}\right)}{d\left(q_{z}^{\prime} D\right)}\right|^{-1} J_{\lambda, \lambda^{\prime}}^{A}
\end{aligned}
$$

and

$$
\begin{aligned}
J_{\lambda, \lambda^{\prime}}^{A}= & \sum_{i j m n}^{6} a_{\lambda, i} a_{\lambda^{\prime}, j}^{*} a_{\lambda, m}^{*} a_{\lambda^{\prime}, n}\left(\mathbf{e}_{\lambda, i}^{A} \cdot \mathbf{e}_{\lambda^{\prime}, j}^{A *}\right)\left(\mathbf{e}_{\lambda, m}^{A *} \cdot \mathbf{e}_{\lambda^{\prime}, n}^{A}\right) \\
& \times \frac{\exp \left[i\left(k_{\lambda, i}^{A}-k_{\lambda^{\prime}, j}^{A *}-k_{\lambda, m}^{A *}+k_{\lambda^{\prime}, n}^{A}\right) d_{A}\right]-1}{i\left(k_{\lambda, i}^{A}-k_{\lambda^{\prime}, j}^{A *}-k_{\lambda, m}^{A *}+k_{\lambda^{\prime}, n}^{A}\right) d_{A}}
\end{aligned}
$$

In the above equations $I_{A}\left(\omega_{\lambda}\right)$ is a dimensionless quantity, $a_{\lambda, l}$ and $a_{\lambda, l+3}(l=1-3)$ stand for the transmission and reflection coefficients $t_{\lambda, l}^{A}$ and $r_{\lambda, l}^{A}$, respectively, $\mathbf{e}_{\lambda, l+3}^{A}=\widetilde{\mathbf{e}}_{\lambda, l}^{A}$, $k_{\lambda, l+3}^{A}=-k_{\lambda, l}^{A}$, and $v_{0}$ is a velocity used for the normalization. If the materials $A$ and $B$ are the same, Eq. (17) reduces to $1 / 2$ of the isotope scattering rate of phonons in the bulk $A$ material, i.e., $\tau_{\text {bulk }}^{-1}=V_{0}^{A} g_{A} \omega^{4} / 4 \pi\left\langle v_{A}{ }^{3}\right\rangle,{ }^{4}$ where $v_{A}$ is the sound velocity and \langle\rangle means the average over three bulk phonon modes.

Figures 1(a) and 1(b) depict the band structures of both the sagittal $(\mathrm{SG})$ and pure transverse $\mathrm{SH}$ phonons in the AlAs/GaAs superlattice with $d_{A l A s}=d_{G a A s}$. Due to the small acoustic mismatch between AlAs and GaAs, the frequency gaps are rather small. It should be noted that the lowest band edges of both the SG and SH modes, i.e., the edges of transverse phonons, coincide with each other.

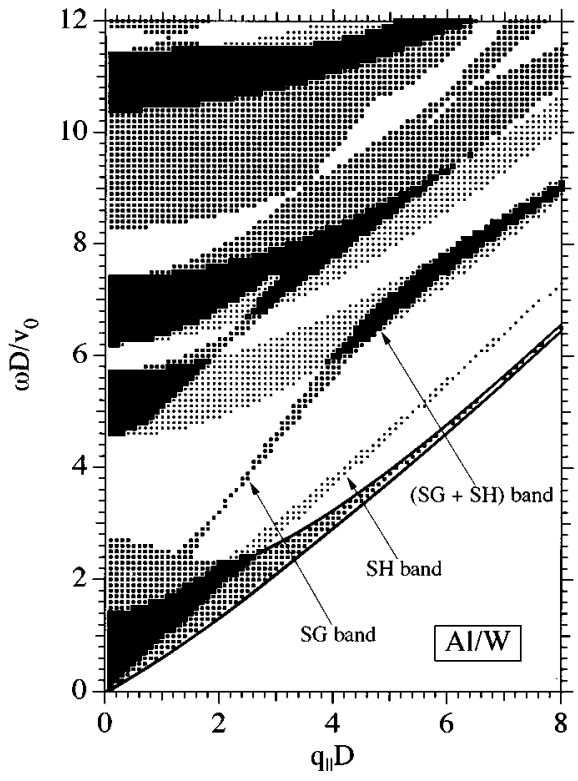

FIG. 2. Band structure of phonons in the Al/W superlattice with $d_{A l}=d_{W}$. Frequency bands due to the SG and SH modes are represented by the large and small dots, respectively. The simultaneous frequency bands of SG and SH modes are filled in black. Inside the band surrounded by the solid lines and the boundary of the simultaneous band the waves are evanescent (or localized) in the growth direction in both the $\mathrm{Al}$ and $\mathrm{W}$ layers (ST band). The numerical values of the sound velocities used are $6.25 \times 10^{5}$ and $5.21 \times 10^{5}$ $\mathrm{cm} \mathrm{s}^{-1}$ ( $L$ modes) and $3.21 \times 10^{5}$ and $2.88 \times 10^{5} \mathrm{~cm} \mathrm{~s}^{-1}(T$ modes) for aluminum and tungsten, respectively and the mass densities are $2.73 \mathrm{~g} \mathrm{~cm}^{-3}$ for aluminum and $19.3 \mathrm{~g} \mathrm{~cm}^{-3}$ for tungsten. $v_{0}$ is the transverse sound velocity $\left(3.21 \times 10^{5} \mathrm{~cm} \mathrm{~s}^{-1}\right)$ in aluminum.

Figure 2 also shows the band structure of the Al/W superlattice with $d_{A l}=d_{W}$. For the $\mathrm{Al} / \mathrm{W}$ superlattice the frequency gaps are much wider than those of the AlAs/GaAs superlattice, so we have plotted the frequency bands of the SG and SH modes simultaneously. An interesting feature of the band structure of the $\mathrm{Al} / \mathrm{W}$ superlattice is the fact that there exists an allowed frequency band below the lowest $\mathrm{SH}$ band, though no such frequency band is present for the AlAs/GaAs superlattice. This extra band in the $\mathrm{Al} / \mathrm{W}$ superlattice (surrounded by the solid lines in Fig. 2) stems from the phonons localized at the interfaces between the tungsten and aluminum, i.e., $k_{\lambda, l}^{A}$ and $k_{\lambda, l}^{B}$ are complex numbers. Owing to the mutual overlapping of the localized lattice displacements along the growth direction due to the finite thicknesses of the constituent layers, these phonons are extended over the superlattice and forms a frequency band. In the following we call this frequency band the Stoneley (ST) band. This is also the origin of the enhanced phonon density of states (DOS) in the Al/W system at low frequencies, which can be seen below. Note that the phonons localized at the layer interfaces are reduced to the Stoneley interface phonons when the layer thicknesses become infinite, which is characteristic of the special combinations of the elastic constants and mass densities of the constituent materials. ${ }^{8}$ No such localized mode exists at the interfaces between bulk GaAs and AlAs. 


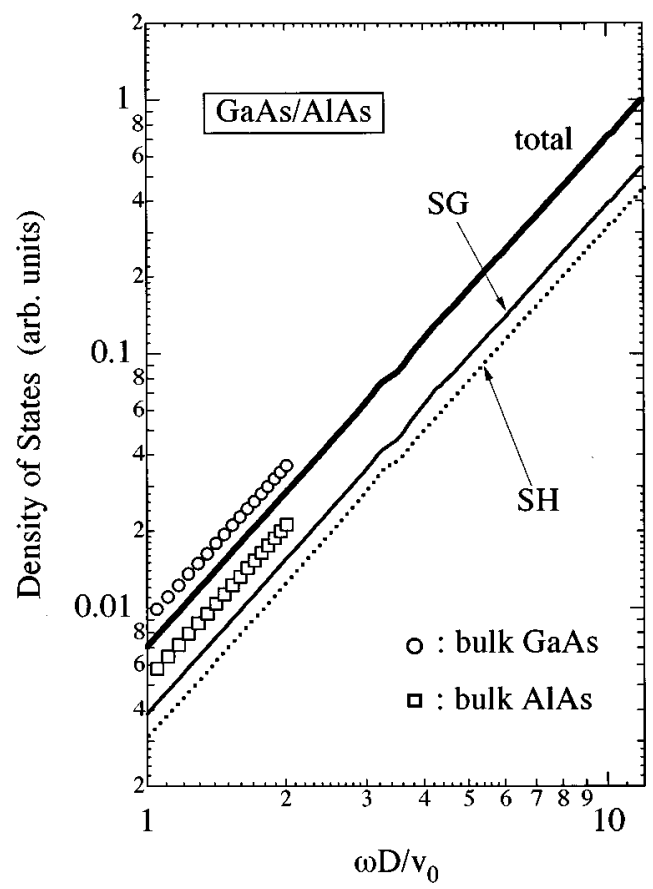

FIG. 3. Density of states (DOS) of phonons in the AlAs/GaAs superlattice corresponding to the band structure of Fig. 1. The bold line is the sum of the SG and SH phonons, represented by the thin solid line and dotted line, respectively. The DOS's of the phonons in the bulk GaAs and AlAs, which are proportional to $\omega^{2}$ (valid in the continuum approximation), are also shown by the circles and squares, respectively.

The DOS of superlattice phonons

$$
G(\omega)=\sum_{\lambda} \delta\left(\omega-\omega_{\lambda}\right)
$$

which reflects the band structures together with those of bulk materials, are plotted in Figs. 3 and 4 for the AlAs/GaAs and $\mathrm{Al} / \mathrm{W}$ superlattices, respectively. At low frequencies the DOS grows in proportion to $\omega^{2}$ as in the bulk materials and for the AlAs/GaAs system the magnitude takes almost the averaged value of the DOS's of bulk AlAs (squares) and GaAs (circles). This means that the magnitude of DOS in the low-frequency region of the $\mathrm{AlAs} / \mathrm{GaAs}$ superlattice is consistent with the one estimated from the formula for the bulk phonons with the mass and elastic constants averaged over GaAs and AlAs. However, for the $\mathrm{Al} / \mathrm{W}$ superlattice the magnitude of DOS at low frequencies is much larger than those of the bulk aluminum (squares) and tungsten (circles). This enhanced DOS of the $\mathrm{Al} / \mathrm{W}$ superlattice is due to the existence of the extra ST band below the lowest band of the transverse phonons as stated above.

At high frequencies the situation is somewhat different. In particular, we see in Fig. 4 for the Al/W superlattice that the DOS increases linearly with $\omega$ in certain frequency ranges and also exhibits various structures. (The frequencies at which singularities are found in the DOS correspond well to the local maxima and minima of the frequency bands shown in Fig. 2.) This linear behavior of the DOS reflects the local-

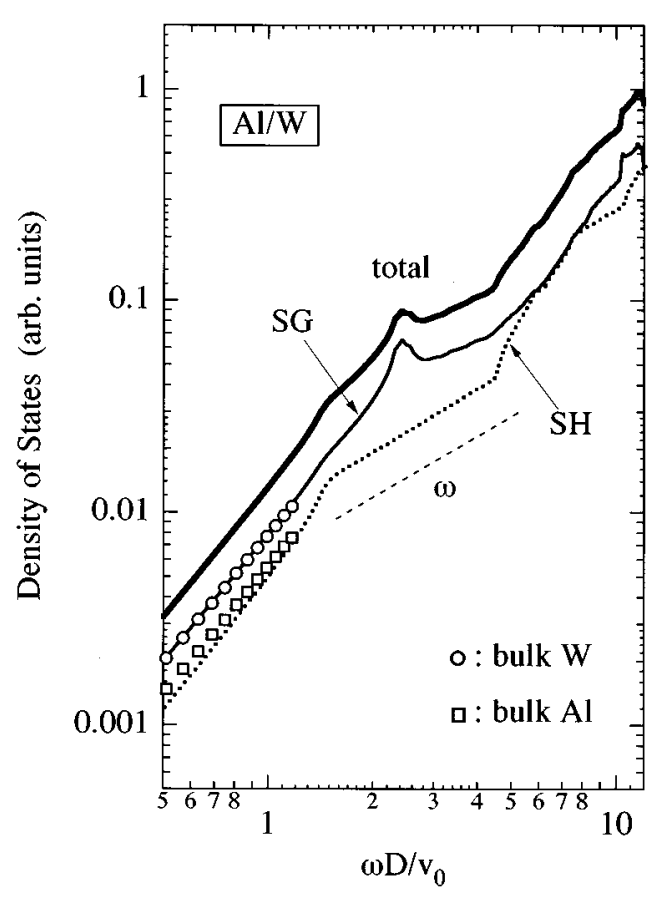

FIG. 4. Density of states (DOS) of phonons in the Al/W superlattice corresponding to Fig. 2. The bold line is the sum of the SG and $\mathrm{SH}$ phonons represented by the thin solid line and dotted line, respectively. Circles and squares are the DOS's of the phonons (proportional to $\omega^{2}$ ) in bulk tungsten and aluminum.

ized (low-dimensional) nature of the phonons in the superlattice, which is shown as narrow lines in the band structures of Fig. 2. Quite unlike the DOS in the Al/W superlattice, the DOS in the AlAs/GaAs superlattice exhibits almost $\omega^{2}$ behavior below $\omega D / v_{0}<3.5$ except for the tiny structures around $\omega D / v_{0}=3.5$ and 10 .

In Fig. 5 we have plotted the isotope scattering rates of $L$ and $T$ phonons propagating along the growth direction of the AlAs/GaAs superlattice together with the corresponding phonon dispersion relations. (The scattering does not exist in the frequency gaps where phonon propagation is prohibited.) Due to the structural anisotropy of the superlattice, the scattering rate depends on both the phonon mode and propagation direction. For comparison, the isotope scattering rate (proportional to $\omega^{4}$ ) in bulk GaAs, which is independent of the phonon polarization and propagation direction, is represented by the dotted line. As expected, the scattering rates are smaller than that in the bulk GaAs because the scattering does not occur in the AlAs layers. We also see that the scattering rate of the $L$ phonons is smaller than that of the $T$ phonons. This can be understood as follows. For phonons in a frequency band the propagation angles in each layer measured from the growth direction are allowed from $0^{\circ}$ to $90^{\circ}$ for the $L$ mode but $0^{\circ}$ to some angle smaller than $90^{\circ}$ for the $T$ mode. Hence the inner products of the polarization vectors between the initial (propagating at $0^{\circ}$ ) and a scattered sagittal phonons [see Eq. (16)] extends from 1 to 0 for the $L$ mode but from 1 to some finite value for the $T$ mode, leading to the enhanced scattering for the transverse phonons.

The similarly enhanced scattering rate of $T$ phonon can be seen in Fig. 6 for the Al/W superlattice at the low-frequency 

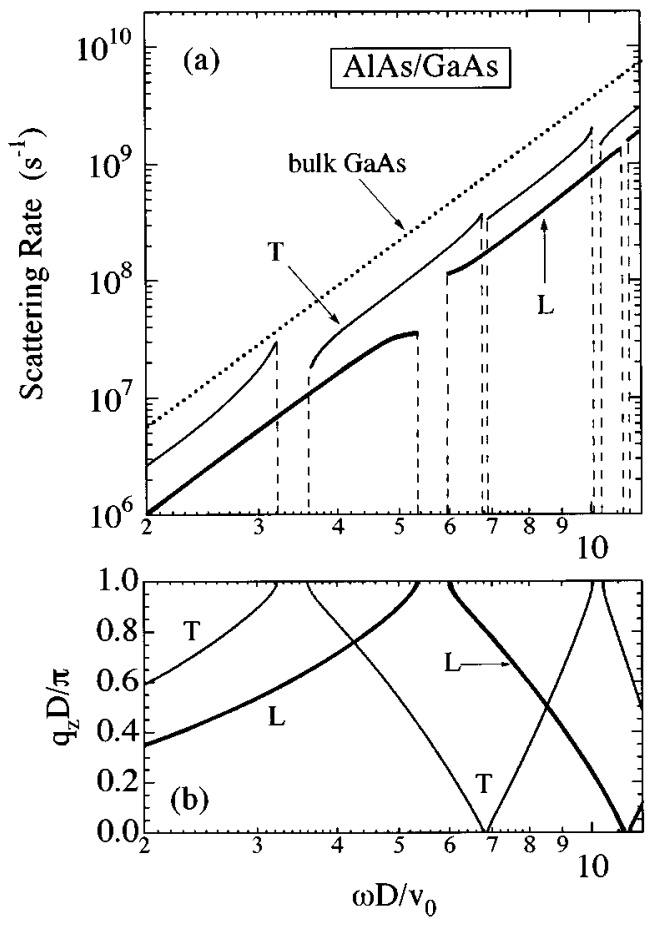

FIG. 5. (a) Isotope scattering rates for longitudinal $(L)$ phonons (bold line) and transverse $(T)$ phonons (thin line) propagating along the growth direction of the $\mathrm{AlAs} / \mathrm{GaAs}$ superlattice with $d_{A l A s}=d_{G a A s}$ and $D=1 \mathrm{~nm}$. [If $D=10 \mathrm{~nm}$ is chosen, the magnitude of the scattering rate should be reduced by a factor of $10^{4}$ without changing the scale of the abscissa as indicated by Eqs. (17)-(19)]. The scattering rate of the phonons in bulk GaAs, proportional to $\omega^{4}$, is also shown by the dashed line. For comparison the phonon dispersion relations for normal propagation are plotted in (b). $v_{0}$ is the transverse sound velocity $\left(3.03 \times 10^{5} \mathrm{~cm} \mathrm{~s}^{-1}\right)$ in GaAs.

region below the lowest-frequency gap. However, the reason for the suppressed $L$ phonon scattering rate is somewhat different from that for the AlAs/GaAs superlattice. The $L$ phonon in this superlattice is scattered predominantly into the ST phonons, but the $T$ phonon is scattered into both the SG and SH phonons, which have larger DOS than that of ST phonons. This is also the reason that we cannot see discernible structures in the scattering rate of $L$ phonon at about $\omega D / v_{0}=2.4$. At higher frequencies very complicated band structures of phonons in the Al/W superlattice makes it hard to tell which scattering rate is larger. Also we note that the scattering rate of low-frequency $T$ phonons is close to the scattering rate of phonons in bulk tungsten. This enhancement is again due to the existence of the ST band below the $\mathrm{SH}$ band.

Here we should note that the $\mathrm{Al} / \mathrm{W}$ superlattice is metallic and hence the electron-phonon scattering should dominate the isotope scattering of phonons. The relaxation times of phonons in the bulk tungsten and aluminum due to electron scattering have been reported at frequencies higher than 0.1 $\mathrm{GHz}$ where the attenuation depends linearly on the frequency. ${ }^{9}$ In this regime the longitudinal phonon relaxation rate in the [001] direction is $\tau_{e l-p h, I}^{-1}=\alpha_{I} \nu$, where $\alpha_{W}=3.3 \times 10^{-3}$ for tungsten ${ }^{10,11}$ and $\alpha_{A l}=2.7 \times 10^{-2}$ for aluminum. ${ }^{12}$ An enhanced attenuation in a metallic superlattice has also been reported. ${ }^{13}$ These results indeed indicate
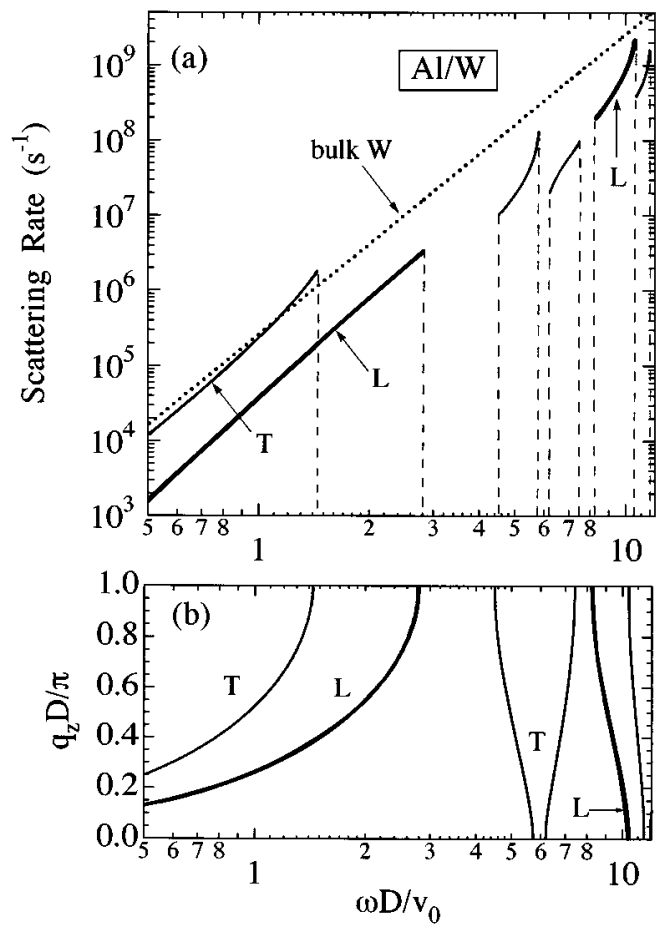

FIG. 6. (a) Isotope scattering rates of the longitudinal $(L)$ phonons (bold line) and transverse $(T)$ phonons (thin line) propagating along the growth direction of $\mathrm{Al} / \mathrm{W}$ superlattice with $d_{A l}=d_{W}$ and $D=1 \mathrm{~nm}$. [If $D=10 \mathrm{~nm}$ is chosen, the magnitude of the scattering rate should be reduced by a factor of $10^{4}$ without changing the scale of the abscissa as indicated by Eqs. (17)-(19)]. The scattering rate of the phonons in bulk tungsten, proportional to $\omega^{4}$, is also shown by the dashed line. For comparison the phonon dispersion relations for normal propagation are plotted in (b). $v_{0}$ is the transverse sound velocity $\left(3.21 \times 10^{5} \mathrm{~cm} \mathrm{~s}^{-1}\right)$ in aluminum.

much larger phonon attenuation due to the scattering with electrons in the gigahertz frequency range. The reason that we have given the numerical calculation for the $\mathrm{Al} / \mathrm{W}$ superlattice is, as remarked repeatedly, to emphasize the existence of the ST band and its effect on the isotope scattering of phonons. Such a ST band and the associated enhancement of the isotope scattering should be also found in nonmetallic (possibly strained) superlattices with a large acoustic mistamch.

To summarize, we have derived a formula for the isotope scattering of phonons in superlattices and also given numerical results for the $\mathrm{AlAs} / \mathrm{GaAs}$ and $\mathrm{Al} / \mathrm{W}$ superlattices. For phonons propagating normal to the layer interfaces, the scattering of the longitudinal phonons in the AlAs/GaAs superlattice (also at the low-frequency region of the $\mathrm{Al} / \mathrm{W}$ superlattice) is smaller than that of the transverse phonons by a factor of 2 or more. Thus, as far as the lattice thermal conductivity in the elastic scattering dominated regime is concerned, we expect that the longitudinal phonons play a more important role in superlattices than in bulk materials.

The author would like to thank H. J. Maris for useful discussions and A. G. Every for helpful comments on the manuscript. This work was supported in part by a Grant-in-Aid for Scientific Research from the Ministry of Education, Science and Culture of Japan (Grant No. 09640385). 
${ }^{1}$ See, for example, M. G. Cottam and D. R. Tilley, Introduction to Surface and Superlattice Excitations (Cambridge University Press, Cambridge, 1989); J. Sapriel and B. D. Rouhani, Surf. Sci. Rep. 10, 1189 (1989).

${ }^{2}$ W. S. Capinski and H. J. Maris, Physica B 219\&220, 699 (1996).

${ }^{3}$ S. Y. Ren and J. D. Dow, Phys. Rev. B 25, 3750 (1982).

${ }^{4}$ For the scattering of bulk phonons see S. Tamura, Phys. Rev. B 27, 858 (1983); 30, 849 (1984).

${ }^{5}$ P. Carruthers, Rev. Mod. Phys. 33, 92 (1961).

${ }^{6}$ S. Tamura, D. C. Hurley, and J. P. Wolfe, Phys. Rev. B 38, 1427 (1988).

${ }^{7}$ R. E. Camley, B. Djafari-Rouhani, L. Dobrzynski, and A. A.
Maradudin, Phys. Rev. B 27, 7318 (1983); B. Djafari-Rouhani, L. Dobrzynski, O. Hardouin Duparc, R. E. Camley, and A. A. Maradudin, ibid. 28, 1711 (1983).

${ }^{8}$ B. A. Auld, Acoustic Fields and Waves in Solids (Krieger, Malabar, FL, 1990), Vol. II.

${ }^{9}$ A. B. Pippard, Proc. R. Soc. London, Ser. A 257, 165 (1960).

${ }^{10}$ C. K. Jones and J. A. Rayne, Phys. Lett. 13, 282 (1964).

${ }^{11}$ M. J. G. Lee, J. M. Perz, and J. Plotnick, Phys. Rev. Lett. 48, 30 (1982).

${ }^{12}$ K. C. Hepfer and J. A. Rayne, Phys. Rev. B 4, 1050 (1971).

${ }^{13}$ W. Chen, Y. Lu, H. J. Maris, and G. Xiao, Phys. Rev. B 50, 14506 (1994). 